\title{
Dual-band Planar Bowtie Monopole for a Fall-Detection Radar and Telemetry System
}

\author{
Ping Jack Soh, Student Member, IEEE, Marco Mercuri, Student Member, IEEE, Gokarna Pandey, Guy \\ A.E. Vandenbosch, Fellow, IEEE, Dominique M.M.-P Schreurs, Fellow, IEEE
}

\begin{abstract}
A dual-band planar bowtie monopole for a falldetection telemetry radar system is presented. Unidirectionality is successfully enabled by a full ground plane. A compact radiator footprint is achieved by closely spacing two bowtie elements for transmit-receive operation, combined with a simple and effective technique for mutual-coupling reduction. The radar antenna shows target location and speed detection capabilities of up to $4 \mathrm{~m}$ with resolution of $30 \mathrm{~cm}$ and fall detection success rate of $95 \%$. Its telemetry capability is then validated practically by frame transmissions through this antenna, which are successfully received by a base station located $5 \mathrm{~m}$ away.
\end{abstract}

Index Terms - Radar antenna, biomedical telemetry, biomedical applications of electromagnetic radiation.

\section{INTRODUCTION}

$\mathrm{A}$ CCIDENTAL falls are the leading cause of injuries and consequently the primary reason of accidental deaths for home-bound patients aged over 65 years [1]. Victims who remain undetected for more than an hour are likely to suffer from prolonged medical complications, with half of them dying within the next six months. Thus, it is imperative that a continuous medical monitoring is provided to enable the prompt dispatching of medical assistance. Although such system could be realized through various sensor-integrated monitoring vests [2], this solution is only effective when consistently worn, whereas conventional methods such as emergency press-buttons are rendered useless once the target is unconscious. To provide a consistent balance between privacy and monitoring effectiveness, a remote fall-detection radar installed indoors is considered most ideal.

The proposed system, realized based on a steppedfrequency continuous wave (SFCW) radar [1], requires a set of compact, high-gain and unidirectional radiators to determine target location and activities (sitting, walking, standing, falling, etc.). A higher gain enables an optimal

Manuscript received October 14, 2012. This work was supported in part by the Malaysian Ministry of Higher Education (MOHE).

P. J. Soh, M. Mercuri, G. Pandey, G. A. E. Vandenbosch and D. M. M.-P. Schreurs are with ESAT-TELEMIC Research Division, Department of Electrical Engineering, Katholike Universiteit Leuven, Kasteelpark Arenberg 10, 3001 Leuven, Belgium. (Phone: +32(0)1632 1150; fax: +32(0)1632 1986; e-mail: pingjack.soh@ esat.kuleuven.be).

P. J. Soh is also with the School of Computer and Communication Engineering, Universiti Malaysia Perlis (UniMAP), 02000 Kuala Perlis, Perlis, Malaysia. (e-mail: pjsoh@unimap.edu.my). transmit power combined with a guaranteed reception of target-reflected signals containing location and movement information. The simultaneous unidirectional and wideband requirements are challenging for antenna designers, as a full ground plane limits antenna bandwidth. Secondly, to avoid a more complex and more lossy RF design (e.g., circulator, divider) resulting from a single transmit-receive radar antenna, the combination of two elements for the same purpose translates into a larger radiator footprint. To maintain compactness, both elements are required to be closely-spaced, which gives rise to high mutual-coupling.

Prior researchers have tried to solve this by using various approaches, e.g., absorbers [3], perfectly conducting boxes [46] and electromagnetic bandgap (EBG) reflectors [7]. Since our radar system requires operation at $2.4 \mathrm{GHz}$ for telemetry, Doppler speed detection at $5.8 \mathrm{GHz}$, and target localization between 6 and $6.5 \mathrm{GHz}$, any additional radiator bandwidth will cause noise pickup and incur additional hardware for signal filtering and amplification.

This work presents a unidirectional antenna array using coplanar waveguide (CPW) bowties, operating in all three target frequency ranges. The prime objective is to satisfy the radartelemetry system's requirements and to overcome foreseen complexities of back-radiation and mutual-coupling. The antenna features a mutual-coupling and back-radiation suppression technique which is compact, effective, and easy to implement, in contrary to the methods presented in [3-5]. Its full capabilities are demonstrated through evaluations in a realistic indoor environment.

\section{RADAR SYSTEM OVERVIEW}

The proposed radar system aims to collect target movement and distance information up to $4 \mathrm{~m}$ distance and with $30 \mathrm{~cm}$ resolution. The block diagram is shown in Fig. 1, consisting of a radar module, a Zigbee module, and a microcontroller. The radar module is custom-built using off-the-shelf RF components: switch, power divider, phase locked-loop (PLL), integrated voltage-controlled oscillator (VCO), low noise amplifier (LNA), and gain block. Signal down-conversion to baseband is performed using an In-phase-Quadrature (IQ) mixer. Both information channels are simultaneously filtered and amplified prior to digitization by the Analogue to Digital Converter (ADC).

The synthesizer in the PLL is programmed by the 
microcontroller to generate the custom 6-6.5 $\mathrm{GHz} \mathrm{SFCW}$ excitation signal. Transmission is preceded by a single-tone $5.8 \mathrm{GHz}$ signal for Doppler speed detection. Both signals are alternated to continuously determine the target's speed and absolute distance. The RF switch connects the antenna alternately to the SFCW transmitter and the Zigbee module. The subject 's activity (sitting, walking, falling, etc.) is then determined through a movement classification algorithm based on machine learning techniques as described in [8].

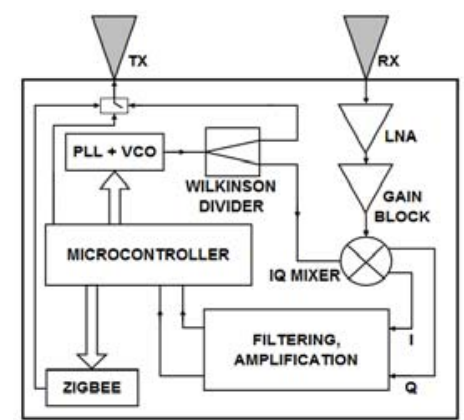

Fig. 1. Block diagram of the radar sensor consisting of radar module, Zigbee module, and microcontroller.

\section{ANTENNA REQUIREMENTS}

The allowable mean Equivalent Isotropically Radiated Power (EIRP) is limited to $-41.3 \mathrm{dBm} / \mathrm{MHz}$ (between 6 and $8.5 \mathrm{GHz}$ ) in order to meet European Telecommunications Standards Institute (ETSI) and Federal Communications Commission (FCC) UWB spectral masks. This makes the unidirectional radiation requirement vital to ensure sufficient received power after reflection and absorption by the subject. Besides reducing power efficiency, the existence of backradiation will also limit the radar's detectable range due to the reduced directivity. To avoid this, a full ground plane is utilized to effectively suppress back radiation, resulting in a thicker radiator. Furthermore, the miniaturized footprint objective results in a small spacing between transmit and receive antennas, giving rise to mutual coupling. This is solved with a vertical wall. At least $5 \mathrm{~dB}$ antenna gain and ca. $80^{\circ}$ half-power beam-width (HPBW) is required for a $4 \mathrm{~m}$ detection distance within $\pm 30^{\circ}$ from the setup. In this way full coverage of a room is reached with a minimum number of such radar setups.

\section{Antenna Design and Evaluations}

The bowtie is designed on a Rogers $\mathrm{RO} 4003$ substrate with a relative permittivity $\left(\varepsilon_{\mathrm{r}}\right)$ of 3.3 , loss tangent $(\tan \delta)$ of 0.0012 and substrate thickness $\left(h_{s}\right)$ of $1.524 \mathrm{~mm}$. It consists of two triangular slots, sized at $T_{\mathrm{W} 1} \times T_{\mathrm{L}}$ and $T_{\mathrm{W} 2}$. A $50 \Omega$ co-planar waveguide (CPW) feed line sized at $F_{\mathrm{L}}$ x $F_{\mathrm{W}} \times F_{\mathrm{G}}$ is centered between both elements. This layer is then secured on the reverse side to a $h_{\mathrm{f}}=18.5 \mathrm{~mm}$ thick foam substrate $\left(\varepsilon_{\mathrm{r}}=1.05\right)$ before attachment to a $h_{\mathrm{c}}=1 \mathrm{~mm}$ thick ground plane. The radiator, substrate and ground plane sizes are standardized to $S_{\mathrm{W}} \times S_{\mathrm{L}}$ to ease fabrication. Dimensions of this antenna are estimated using [9]:

$$
\begin{aligned}
& 2 S_{W}+2 W_{s}+W_{t}=1.6 \lambda_{0} / \sqrt{\varepsilon_{r}} \\
& T_{W 1}=0.5 \lambda_{0} / \sqrt{\varepsilon_{r}}
\end{aligned}
$$

where $\lambda_{0}$ is the wavelength at $2.4 \mathrm{GHz}$. Calculations using these equations yields $110.10 \mathrm{~mm}$ and $34.41 \mathrm{~mm}$, respectively. They are then optimized to be $T_{\mathrm{W} 1}=20 ; T_{\mathrm{W} 2}=$ $2.4 ; T_{\mathrm{L}}=14.7 ; F_{\mathrm{L}}=24 ; F_{\mathrm{W}}=3.5 ; F_{\mathrm{G}}=0.6 ; S_{\mathrm{W}}=36$; and $S_{\mathrm{L}}=$ 42, all in mm, as given in Fig. 2.

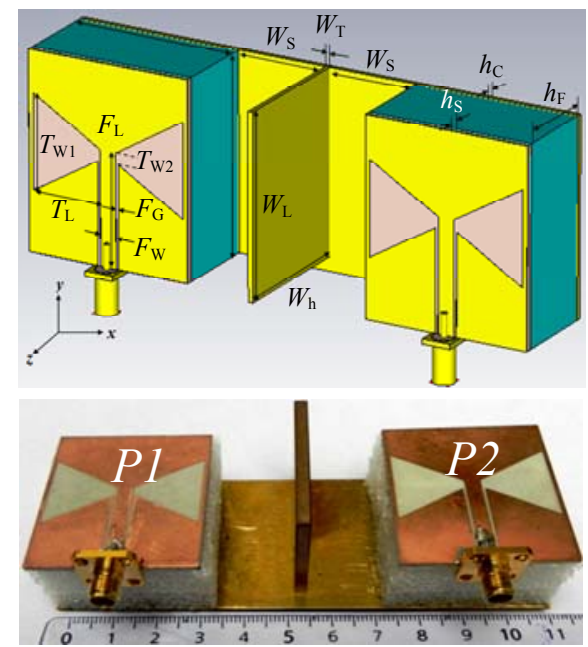

Fig. 2. Topology of the bow-tie array: dimensions (top), prototype (bottom).

Simulations and measurements for the two realizations of the prototype (named $P 1$ and $P 2$ ) agree very well (Fig. 3). A lower band $(B 1)$ centered at $f_{\mathrm{c}}=2.49 \mathrm{GHz}$ and an upper band (B2) centered at $f_{\mathrm{c}}=6.1 \mathrm{GHz}$ are seen. The bandwidths $(B W)$ produced are $B 1=185 \mathrm{MHz}$ and $B 2=$ ca. $865 \mathrm{MHz}$, taken at the $-10 \mathrm{~dB}$ reflection coefficient $\left(S_{11}\right)$ borders. The small measurement discrepancies are due to the difficulties in producing an exactly-dimensioned foam substrate. Cutting inaccuracies also result in an uneven foam surface and consequently the existence of air gaps in the dielectric-tofoam layer. A summary of the evaluations is listed in Table I.

For radar operation and speed detection, the two antenna elements are secured on a common ground plane of size ca. $0.90 \lambda_{0} \times 0.34 \lambda_{0}$, see Fig. 2 . Due to the close spacing $\left(0.32 \lambda_{0}\right)$ a high mutual-coupling $\left(S_{21}\right)$ of at least $-25 \mathrm{~dB}$ is observed in Fig. 3. Disagreement between simulated and measured $S_{21}$ is observed, believed to be caused by the foam's loss tangent exclusion from the simulated model. The initially high measured $S_{21}$ is then solved by centering a vertical wall sized at $W_{\mathrm{L}} \times W_{\mathrm{h}}=39 \times 31 \mathrm{~mm}^{2}\left(\right.$ ca. $0.31 \lambda_{0} \times 0.25 \lambda_{0}$ ) between the two antennas. The spacing of $W_{\mathrm{S}}=19.5 \mathrm{~mm}$ results in more than $10 \mathrm{~dB} S_{21}$ reduction in $B 2$, with a simultaneous $B W$ reduction at $B 2$ 's lower band edge. This is due to the structure's lower Q with the elimination of capacitive coupling. Nonetheless, $B W$ at $B 2$ is still sufficient to provide proper radar operation.

Radiation patterns and gains have been measured in an anechoic chamber at three frequencies, $f_{1}=2.45 \mathrm{GHz}, f_{2}=5.8$ 
$\mathrm{GHz}$ and $f_{3}=6.5 \mathrm{GHz}$, see Fig. 4. Measurements for both $P 1$ and $P 2$ agree very well. A HPBW difference of less than $8^{\circ}$ is observed between the two antennas. Sidelobes at $f_{1}$ in both Eand H-planes exist, enabling node-to-node Zigbee communication between two radar units when mounted on walls and ceilings in the same room. In agreement with the simulations, slight pattern rotations at $f_{2}$ and $f_{3}$ are observed in the H-plane.

A parametric study has demonstrated that out of the eight antenna parameters, variation of six of them changes $f_{\mathrm{c}}$. The B1 $f_{\mathrm{c}}$ can be adjusted by changing the substrate size $\left(S_{\mathrm{W}}\right.$ and $\left.S_{\mathrm{L}}\right)$. The B2 $f_{\mathrm{c}}$ can be modified by changing the feed dimensions $\left(F_{\mathrm{W}}, F_{\mathrm{G}}\right.$ and $\left.F_{\mathrm{L}}\right)$ and $T_{\mathrm{L}}$. Changes to $T_{\mathrm{W} 1}$ and $T_{\mathrm{W} 2}$ alter the antenna input impedance while maintaining $f_{\mathrm{c}}$ in both B1 and B2. Meanwhile, the HPBWs in the E- and H-planes are also tunable by varying $F_{\mathrm{L}}, F_{\mathrm{W}}, S_{\mathrm{L}}$ and $S_{\mathrm{W}}$.

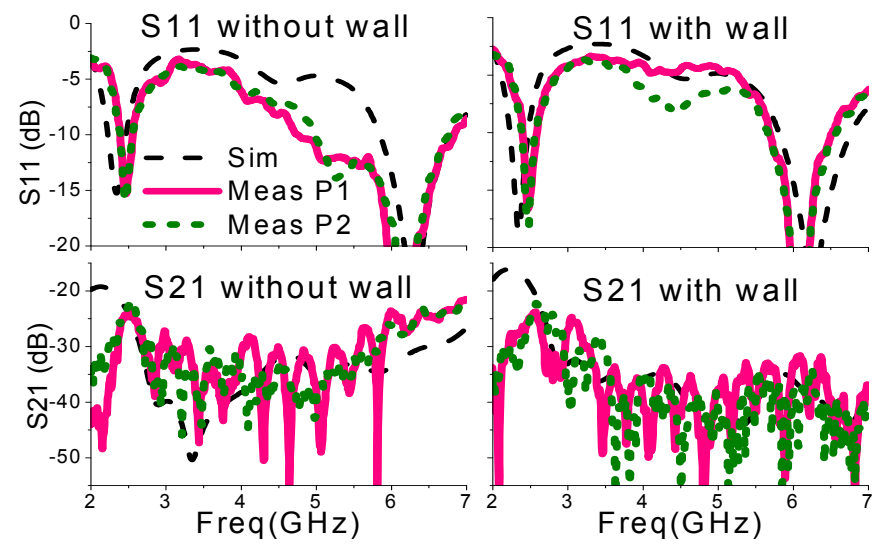

Fig. 3. Simulated and measured $S$-parameters of the elements $P 1$ and $P 2$.

TABLE I. Simulated And Measured Antenna Performance

\begin{tabular}{lccc}
\hline \hline Antenna Parameters & Sim & $P 1$ & $P 2$ \\
\hline$B 1$ Freq $(\mathrm{GHz})$ & $2.22-2.48$ & $2.40-2.59$ & $2.39-2.57$ \\
$B 2 \mathrm{Freq}(\mathrm{GHz})$ & $5.81-6.77$ & $5.69-6.51$ & $5.66-6.57$ \\
$B 1 B W(\mathrm{MHz})$ & 260 & 190 & 180 \\
$B 2 B W(\mathrm{MHz})$ & 960 & 820 & 910 \\
$H P B W\left({ }^{\circ}\right) 2.45 \mathrm{GHz}$ & 60 (Eplane) & 48 (Eplane) & 45 (Eplane) \\
& 41 (Hplane) & 22 (Hplane) & 14 (Hplane) \\
$H P B W\left({ }^{\circ}\right) 5.8 \mathrm{GHz}$ & 63 (Eplane) & 69 (Eplane) & 70 (Eplane) \\
& 50 (Hplane) & 55 (Hplane) & 45 (Hplane) \\
$H P B W\left({ }^{\circ}\right) 6.5 \mathrm{GHz}$ & 71 (Eplane) & 84 (Eplane) & 80 (Eplane) \\
Gain $(\mathrm{dB}) 2.45 \mathrm{GHz}$ & 54 (Hplane) & 59 (Hplane) & 55 (Hplane) \\
Gain $(\mathrm{dB}) 5.8 \mathrm{GHz}$ & 5.7 & 5.2 & 5.0 \\
Gain $(\mathrm{dB}) 6.5 \mathrm{GHz}$ & 8.5 & 7.6 & 8.3 \\
\hline
\end{tabular}

\section{RADAR VALIDATION}

The antenna is integrated with the radar system for evaluation in a realistic indoor environment, (i.e., including chairs, table, etc). Speed and location of the subjects-undertest (SUTs) are first determined, prior to the telemetry link evaluation. The radar setup is secured onto a $1.6 \times 1.35 \mathrm{~m}^{2}$ partition-wall, $1.1 \mathrm{~m}$ from the floor. Distances between 1 and $4 \mathrm{~m}$ away this setup are marked. SUTs include two human subjects as well as an ideally-reflecting $60 \times 40 \mathrm{~cm}^{2}$ metal plate elevated $1.4 \mathrm{~m}$ above the ground. To ensure

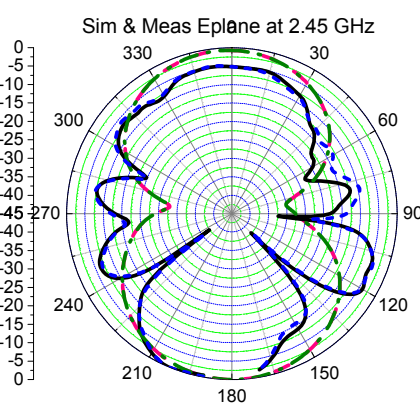

(a)

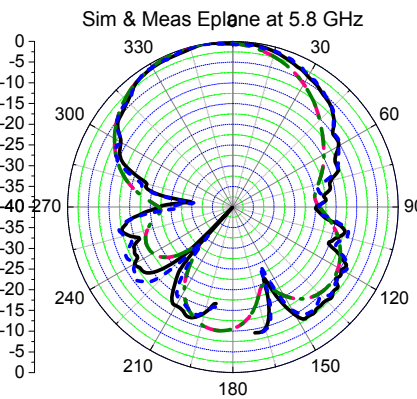

(c)

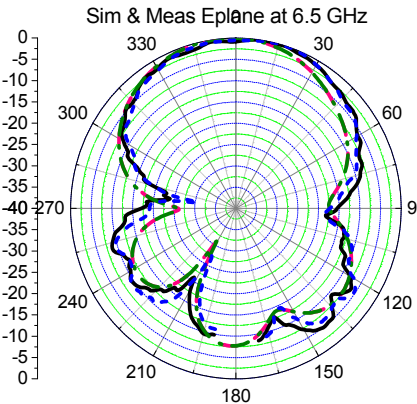

(e)

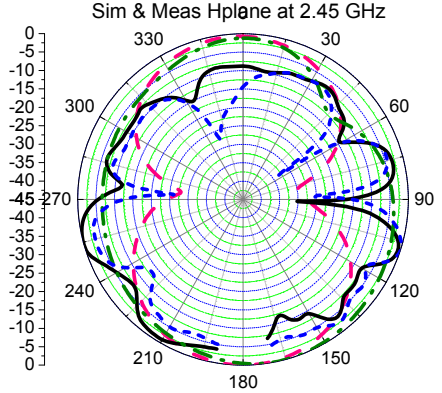

(b)

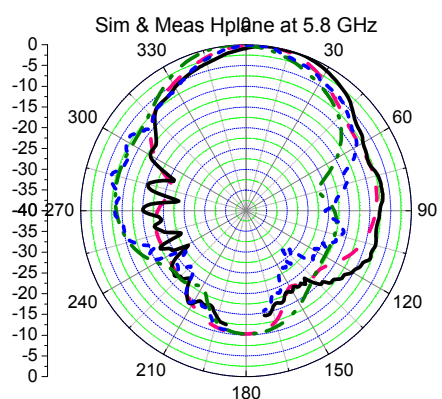

(d)

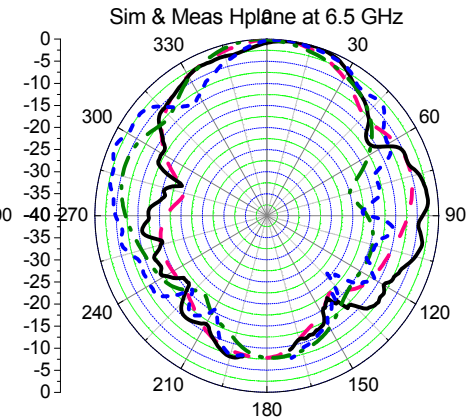

(f)
Fig. 4. Simulated and measured radiation patterns for the array structure: (a) E-plane for $2.45 \mathrm{GHz}$; (b) H-plane for $2.45 \mathrm{GHz}$; (c) E-plane for $5.8 \mathrm{GHz}$; (d) H-plane for $5.8 \mathrm{GHz}$; (e) E-plane for $6.5 \mathrm{GHz}$; and (f) H-plane for 6.5 GHz. Legend: $($ pink-dashed $)=P 1$ sim; $($ black-solid $)=P 1$ meas; $($ greendash-dot $)=P 2$ sim; (blue-short dash $)=P 2$ meas

TABLE II. RADAR EVALUATION USING DIFFERENT SUBJECTS/DISTANCES

\begin{tabular}{lccc}
\hline \hline $\begin{array}{l}\text { Distance }(\mathrm{m}), \text { Stand } \\
\text { angle }\left(^{\circ}\right)\end{array}$ & $\begin{array}{c}\text { Metal plate } \\
\left(0.6 \times 0.4 \mathrm{~m}^{2}\right)\end{array}$ & $\begin{array}{c}\text { Subject 1 } \\
(1.78 \mathrm{~m}, 88 \mathrm{~kg})\end{array}$ & $\begin{array}{c}\text { Subject 2 } \\
(1.78 \mathrm{~m}, 75 \mathrm{~kg})\end{array}$ \\
\hline $1.0,0^{\circ}$ & Yes & Yes & Yes \\
$2.0,0^{\circ}$ & Yes & Yes & Yes \\
$3.0,0^{\circ}$ & Yes & Yes & Yes \\
$3.5,0^{\circ}$ & Yes & Yes & Yes \\
$4.0,0^{\circ}$ & Yes & Yes & Yes \\
$5.0,0^{\circ}$ & Yes & No & No \\
$1.0,30^{\circ}$ & Yes & Yes & Yes \\
$2.0,30^{\circ}$ & Yes & Yes & Yes \\
$3.0,30^{\circ}$ & Yes & No & No \\
$4.0,30^{\circ}$ & No & No & No \\
\hline \hline
\end{tabular}

repeatability, each subject at each location is tested three times. Evaluations yielding three accurate SUT locations are marked as 'Yes', while any failure will result in a 'No'. The results shown in Table II prove that absolute distances for all three SUTs can be determined up to $4 \mathrm{~m}$ at $0^{\circ}$. A sample evaluation result processed using the Inverse Fast Fourier Transform (IFFT) with environmental compensation described in [10] is shown in Fig. 5. When SUTs are located $30^{\circ}$ relative to the radar system, distance-detection capability is lowered to 
$3 \mathrm{~m}$ for the metal plate, and $2 \mathrm{~m}$ for both human subjects. This indicates the relative signal degradation caused by the human body.

The detection distance can be increased for larger indoor environments without increasing transmit power, which is limited by regulatory standards, by first lowering the crosscoupling between the two antennas, and then increasing the receiving baseband gain. In this way, the SUT-reflected signal from beyond $4 \mathrm{~m}$ can then be distinguished by the receiver's ADC. Meanwhile, the current $30 \mathrm{~cm}$ resolution can also be refined by increasing the antenna's operating bandwidth, e.g., doubling the bandwidth from the current $500 \mathrm{MHz}$ to $1 \mathrm{GHz}$ results in a $15 \mathrm{~cm}$ distance resolution instead of $30 \mathrm{~cm}$.

For speed evaluation, five readings are acquired for each SUT and each movement, i.e., during (1) walking ( $1 \mathrm{~m}$ to $4 \mathrm{~m}$ and vice versa), (2) sitting on a chair, and (3) frontal falls at 2, 3 , and $4 \mathrm{~m}$. These speed evaluations reported in [8] clearly show a difference between a fall event and regular walking or sitting, with $95 \%$ accuracy. The distinction is based on the fact that the subject's speed continuously increases and then ends abruptly upon fall completion, whereas during walking or sitting, Doppler signals indicate movements with controlled speeds.

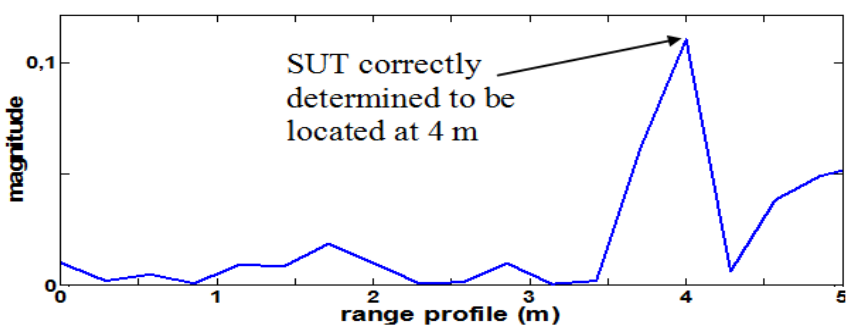

Fig. 5. Measured range profile using IFFT and environment compensation $\lceil 10\rceil$.

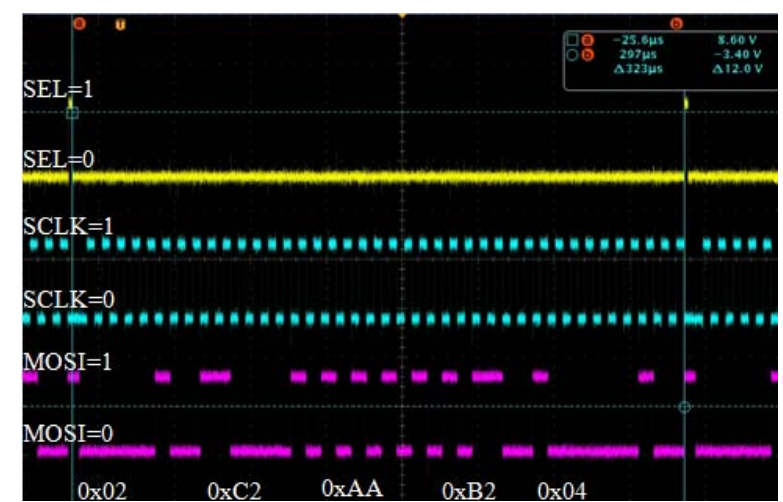

Fig. 6. Signals probed from the receiving Zigbee base station using an oscilloscope for different port signals (from top to bottom): SEL, SCLK and MOSI.

Finally, telemetry has been evaluated by transmitting known frame buffers from the setup to a Zigbee receiving base station located $5 \mathrm{~m}$ away. Three signals from its Serial Peripheral Interface (SPI) bus, i.e. SEL, SCLK and MOSI are read using an oscilloscope. This SPI connects the buffers between the transceiver and microcontroller. SEL $=0$ enables read access, SCLK provides the clock synchronization while
MOSI indicates the data transfer direction (e.g. master-toslave or vice versa). Signals from the receiver module indicated that the transmitted signals have been received without any packet loss or bit errors. A sample of such reading is shown in Fig. 6.

\section{CONCLUSIONS}

A dual-band, unidirectional monopole for a compact indoor fall-detection radar and telemetry system is presented. The unidirectionality is enabled through an optimized full ground plane, and its high mutual coupling due to the small element spacing is reduced by at least $10 \mathrm{~dB}$ using a vertical wall. This antenna is then integrated into the proposed radar system for a comprehensive evaluation, determining speed and absolute subject's location before finally transmitting telemetry data. Besides being electrically small, the unequal bandwidth generated at the lower and higher bands provided for narrowband Zigbee telemetry at $2.45 \mathrm{GHz}$ and wideband radar operation using one antenna setup. Radar measurements indicate the ability to determine a subject's absolute distance up to $4 \mathrm{~m}$ at $0^{\circ}$, while telemetry tests using a Zigbee module indicate an excellent wireless transmission capability.

\section{REFERENCES}

[1] M. Mercuri, D. Schreurs, and P. Leroux, "SFCW Radar for Indoor Fall Detection," in IEEE Topical Conf. on Biomedical Wireless Technologies, Networks and Sensing Systems (BioWireleSS), Jan 15-19, 2012, pp. 53-56.

[2] S. Park and S. Jayaraman, "Enhancing the Quality of Life Through Wearable Technology," IEEE Engineering in Medicine and Biology Mag., vol. 22, no. 3, pp. 41-48, 2003.

[3] E. S. Eide, "Ultra-wideband Transmit/Receive Antenna Pair for Ground Penetrating Radar," IEE Proc. in Microwaves, Antennas and Propagation, vol. 147, no. 3, pp. 231-235, 2000.

[4] F. Sagnard and F. Rejiba, "Wideband Coplanar Waveguide-fed Bowtie Slot Antenna for a Large Range of Ground Penetrating Radar Applications," IET Microwaves, Antennas and Propagation, vol. 5, no. 6, pp. 734-739, 2011.

[5] A. A. Lestari, E. Bharata, A. B. Suksmono, A. Kurniawan, A. G. Yarovoy, and L. P. Ligthart, "A Modified Bow-Tie Antenna for Improved Pulse Radiation," IEEE Trans. on Antennas and Propagation, vol. 58, no. 7, pp. 2184-2192, 2010.

[6] S. Qu, J. Li, Q. Xue, C. H. Chan, and S. Li, "Wideband and Unidirectional Cavity-Backed Folded Triangular Bowtie Antenna," IEEE Trans. on Antennas and Propagation, vol. 57, no. 4, part. 2, pp. 1259-1263, 2009.

[7] Y. Ito, M. Ameya, M. Yamamoto, and T. Nojima, "Unidirectional UWB Array Antenna using Leaf-Shaped Bowtie Elements and Flat Reflector," Electronics Letters, vol. 44, no. 1, pp. 9-11, 2008.

[8] P. Karsmakers, T. Croonenborghs, M. Mercuri, D. Schreurs, and P. Leroux, "Automatic in-door fall detection based on microwave radar measurements," European Radar Conference (EuRAD), Oct. 28-Nov 2 2012. - accepted for publication, to appear.

[9] K. Li, C. H. Cheng, T. Matsui and M. Izutsu, "Simulation and Experimental Study on Coplanar Patch and Array Antennas," AsiaPacific Microwave Conference (APMC), Dec 3-6, 2000, pp. 1411-1414.

[10] M. Mercuri, P.J. Soh, L. Boccia, D. Schreurs, G.A.E. Vandenbosch, P. Leroux, and G. Amendola, "Optimized SFCW Radar Sensor Aiming at Fall Detection in a Real Room Environment", IEEE Topical Conference on Biomedical Wireless Technologies (BioWireless), Austin, TX, USA, 20-23 Jan. 2013. - accepted for publication, to appear. 